\title{
A Method Based on Lorenz Disturbance and Variational Mode Decomposition for Wind Speed Prediction
}

\author{
Yagang ZHANG ${ }^{1,3}$, Shuang $\mathrm{GAO}^{1}$, Minghui BAN ${ }^{1}$, Yi SUN ${ }^{2}$ \\ ${ }^{1}$ State Key Laboratory of Alternate Electrical Power System with Renewable Energy Sources, North China \\ Electric Power University, Beijing, 102206, China \\ ${ }^{2}$ Hebei Electric Power Research Institute, Shijiazhuang, Hebei 050022, China \\ ${ }^{3}$ Interdisciplinary Mathematics Institute, University of South Carolina, Columbia, SC 29208, United States \\ yagangzhang@ncepu.edu.cn
}

\begin{abstract}
Wind power is one of the most promising means of power generation. But the time-varying of wind speed is the most fundamental problem for power generation control system. Therefore, accurate wind speed prediction becomes particularly important. However, traditional wind speed predictions often lack consideration of the influence of atmospheric dynamic system. And few papers have introduced VMD method into the field of wind speed prediction. Thus, combined with four neural networks, this paper develops a wind speed prediction method based on Lorenz system and VMD, obtains LD-VMD-Elman wind speed prediction model. Simulation results show that: 1) As for wind speed prediction, Elman neural network has higher prediction accuracy and smaller error. 2) The models which added Lorenz disturbance can describe the actual physical movement of wind more accurately. 3) VMD can abstract the changing rules of different wind speed frequencies to improve the prediction effect. This paper makes up for the lack of consideration of atmospheric dynamic system. The Lorenz equation is used to describe the atmospheric dynamic system, which provides a new thought for wind speed prediction. The LD-VMD-Elman model significantly improves the accuracy of wind speed prediction and contribute to the power dispatch planning.
\end{abstract}

Index Terms - wind speed prediction, atmospheric dynamics system, Lorenz system, artificial neural network.

\section{INTRODUCTION}

In recent years, the energy and environmental problems such as air pollution is getting worse and worse. Unreasonable energy structure is one of the major factors that aggravate environmental problems. Fossil fuels are the most widely used energy source in the world, and their pollution to the environment is obviously serious. Vigorously developing new energy has become the inevitable course for global energy development and transformation. Wind energy, as a huge amount of clean energy, has attracted more and more attention from countries. The rapid development of wind power has led to an increase in wind power proportion in power grid. However, the random volatility of the wind leads to the instability of wind power. If the wind speed can be accurately predicted in advance when the wind power is connected to the grid, the dispatching of the power system

This research was supported partly by the National Natural Science Foundation of China (51637005), the Fundamental Research Funds for the Central Universities (2017MS166) and the Natural Science Foundation of Hebei Province (G2016502009). can be prepared in advance and the dispatching operators can make effective decisions. Therefore, accurate wind speed prediction contributes to the large-scale development and utilization of wind power resources and promotes wind power integration.

Researchers have done a lot of work on wind speed prediction in recent years. The methods are roughly divided into three types: the physical method, the statistical method and the artificial intelligence method [1]. 1) The physical method mainly predict the wind speed through weather information such as terrain, air pressure and temperature. Numerical weather prediction (NWP), as a typical physical model, predicts the distribution of meteorological elements according to the actual atmospheric conditions, certain initial values and boundary conditions [2]. References [3-5] proposed wind speed prediction models based on physical methods. But the study found that physical method is only suitable for ultra-short-term wind speed prediction. 2) Statistical method mainly use historical data, pattern recognition, parameter estimation and model checking to build mathematical models to solve problems. Usually statistical models contain autoregressive models (AR), moving average models (MA), and autoregressive moving average models (ARMA). However, although the ARMA model can describe linear dynamic processes, it is only applicable to stationary random sequences with zero mean. The wind speed time series is non-stationary obviously. Therefore, when building ARMA model for wind speed prediction, the time series must be non-stationary by adding trend and periodicity. References [6] and [7] proposed different wind speed prediction models based on the statistical method. 3) Artificial Intelligence (AI) is a good prediction method developed in recent years. It is considered as one of the three most advanced technologies in the 21st century and has developed rapidly since the 1970s. At present, artificial intelligence technology applied in the field of wind speed prediction mainly includes the artificial neural network (ANN), fuzzy logic, support vector machine (SVM) and so on. References [8-11] proposed ANN-based wind speed prediction models. References [12-14] proposed wind speed prediction models based on the fuzzy logic. References [15-18] introduced wind speed prediction models based on SVM.

Obviously, this classification is not absolute. There are 
few models that use only a single method for wind speed prediction. In order to optimize the prediction process and improve the result accuracy, hybrid models has gradually become popular. Reference [19-25] present wind speed prediction method based on hybrid models.

However, on the one hand, almost all of them only improved the algorithm, but did not consider the influence of the atmospheric dynamic system on the wind and ignored the non-linear characteristics of wind. Atmospheric motion is a deterministic dynamic system and its behavior can be described by a set of Lorenz equations [26]. In fact, the motivation of Lorenz system approach has been mentioned in the author's past papers in this field. Reference [23] and [27] are our previous papers in the field of wind speed prediction. On the other hand, most scholars only use the traditional EMD or EEMD to decompose the wind speed sequence, and there are few papers that use the newly developed VMD which is noise robust to preprocess the wind speed sequence data [28-29]. At the same time, the nonlinear modeling approaches have been developed in recent years. Reference [30-33] listed some of them that have been proved to be effective in various fields. Wind speed series is a kind of nonlinear time series and the neural network has strong ability of nonlinear mapping, it can complete highly complex input and output non-linear mapping, which is incomparable with traditional wind speed prediction methods.

Based on this, this paper proposes a wind speed prediction model based on VMD and Lorenz system. First, a set of Lorenz equations is used to describe the atmospheric dynamic system, and the equation is solved under given initial conditions and parameters to obtain the optimal disturbance coefficient. Secondly, the original wind speed sequence is decomposed into several IMF components by VMD. Then, each IMF is predicted by BP neural network, RBF neural network, Elman neural network and LSTM neural network, respectively. Finally, the predicted values of each IMF are summed up, and the predicted results are modified by the Lorenz optimal disturbance coefficient obtained in the first step, and the LD-VMD-X (X represents the undetermined neural network model) wind speed prediction model is obtained. The single wind speed prediction methods are compared vertically, and the results obtained by combining different neural networks are compared horizontally. Finally, the LD-VMD-Elman wind speed prediction model which get the highest prediction accuracy is obtained. The contributions and innovations of this paper are summarized as follows:

(1) The Lorenz system is introduced into the wind speed prediction model to describe the effect of atmospheric motion on wind speed, which provides a new idea for the field of wind speed prediction.

(2) This paper proposes an innovative wind speed prediction model named LD-VMD-Elman. Compared with other 11 wind speed prediction model, it achieved the highest prediction accuracy on all four data sets.

(3) Using VMD that is noise robust to preprocess the wind speed sequence data, the wind speed volatility is reduced, which is helpful for primary wind speed prediction model to grasp the law of wind speed.

(4) For LD-VMD-X prediction method, by trying different $X$ predictions, LD-VMD-Elman with the highest prediction accuracy is obtained, which provides a new model selection method for the establishment of wind speed prediction model.

The structure of this paper is as follows: section 2 describes the theoretical process of Lorenz system and VMD. Section 3 introduces the establishment process of wind speed prediction model based on VMD and Lorenz, and gives the flow chart of the model establishment. In section 4, the results of the simulation experiment are introduced and the error is analyzed. Section 5 gives the conclusion

\section{LORENZ CHAOTIC SYSTEM AND VMD}

Lorenz chaotic system and VMD are the two important concepts of model proposed in this paper. In this section, we will introduce their theory and deduction process respectively.

\section{A. Lorenz chaotic system}

In 1963, when American meteorologist Edward Lorenz studied the natural convection system under the action of temperature gradient, he calculated the non-periodic phenomenon from the determined equation (later known as Lorentz equation) and found the first chaotic attractor. The Lorenz system is the earliest dissipative system for chaotic motion found in numerical experiments [34-35]. Its state equation (1) (the Lorenz equation) is a simplified model of weather forecasting.

$$
\left.\begin{array}{c}
\dot{x}=-\sigma(x-y) \\
\dot{y}=-x z+r x-y \\
\dot{z}=x y-b z
\end{array}\right\}
$$

Where $x$ denotes the convection motion amplitude, $y$ denotes the horizontal temperature difference between the ascending and descending fluid in the convection, and $Z$ denotes the deviation of the vertical temperature difference caused by convection from the equilibrium state without convection (convective equilibrium). $\sigma, r$ and $b$ are the Prandtl number, the Rayleigh number (or Reynolds number), and the parameter related to the size of the container (representing the range of the microclimate area). When $\sigma=10, b=8 / 3$ and $r=28$, the system appears as chaotic, and its solution is unstable and sensitive to the initial conditions. In fact, such a solution is chaos [26].

In this study, the solution vector in the chaotic state of the Lorenz system is used as the wind speed disturbance sequence, and the obtained disturbance sequence is applied to revise predictions of different models. Figure 1 shows the Realization of Lorenz disturbance sequence.

In figure $1, h_{0}$ denotes the initial condition, $n$ denotes the wind speed number, $\left(x_{k}, y_{k}, z_{k}\right)(k=1,2, \ldots, n)$ are the solvation of Lorenz equations, $x_{n}^{\prime}, y_{n}^{\prime}, z_{n}^{\prime}$ represent the normalized values of three solutions, $x_{\min }, y_{\min }, z_{\min }$ and $x_{\max }, y_{\max }, z_{\max }$ denote minimum value and maximum value of $x, y, z$ respectively, $h_{k}$ indicates $\left(x_{n}^{\prime}, y_{n}^{\prime}, z_{n}^{\prime}\right), D$ denotes Lorenz disturbance sequence that we need. 


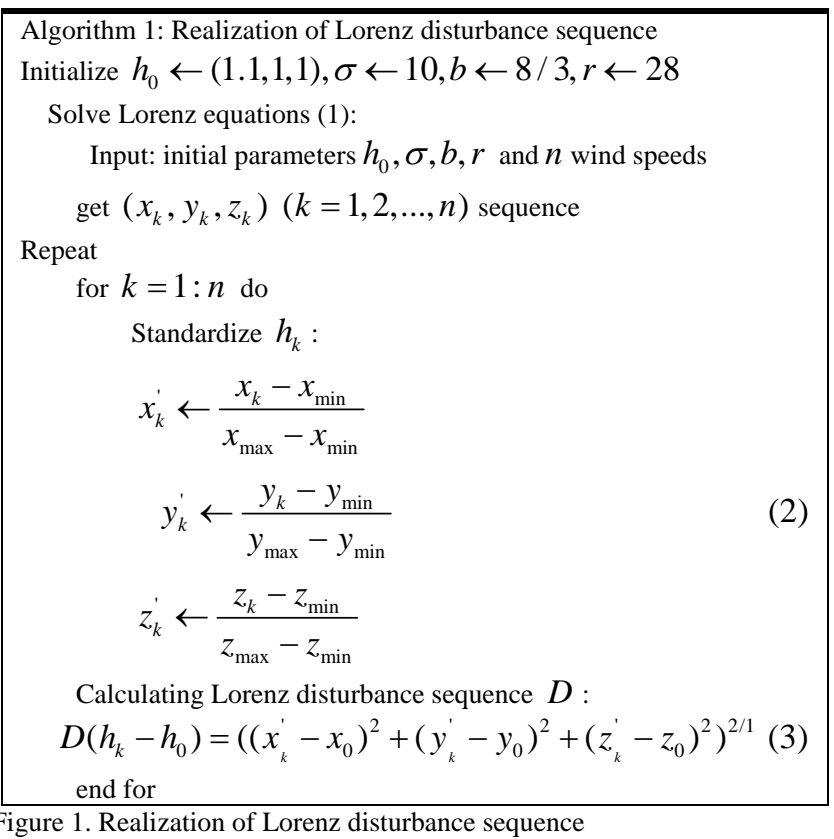

\section{B. Variational mode decomposition}

The wind speed sequence is a non-linear time series, and data preprocessing can effectively improve the prediction accuracy [36]. Variational mode decomposition (VMD) is a signal decomposition method proposed by Dragomiretskiy and Zosso in 2014 [37-38]. It is a new adaptive signal processing technology and has excellent noise immunity. Its performance is better and more stable compared with the ensemble empirical mode decomposition (EEMD) and the empirical mode decomposition (EMD). VMD decomposes the signal into mode $u_{k}$ with different scales. It has specific sparse characteristics while generating the main signal. And assume that each mode $k$ can be concentrated near the center pulsation $\omega_{k}$ determined during the decomposition process.

In order to obtain the mode bandwidth, the following steps should be satisfied: 1) Calculating the analytical signal associated with the modal by Hilberthuang transform to obtain a unilateral spectrum. 2) Adding an exponential term adjusts the center frequency of the modality and shifts the modal spectrum to the baseband. 3) The demodulation signal is processed by H1-Gauss smoothing to estimate the bandwidth. A variational constraint problem is obtained by the above three steps:

$$
\begin{aligned}
\min _{\left\{u_{k}\right\},\left\{\omega_{k}\right\}}\left\{\sum_{k}\left\|\partial_{t}\left[\left(\delta(t)+\frac{j}{\pi t}\right) * u_{k}(t)\right] e^{-j \omega_{k} t}\right\|_{2}^{2}\right\} \\
\text { s.t. } \sum_{k} u_{k}=f(t)
\end{aligned}
$$

Where $f(t)$ denotes the decomposed main signal, $\left\{u_{k}\right\}:=\left\{u_{1}, \ldots, u_{K}\right\}$ and $\left\{\omega_{k}\right\}:=\left\{\omega_{1}, \ldots, \omega_{K}\right\}$ represent the set containing all modes and their center frequencies, respectively, $\delta(t)$ denotes the Dirac distribution, * denotes the convolution.

Then, we add the quadratic penalty term $\alpha$ and the Lagrange multiplier $\lambda$ to make the problem unconstrained. The combination can benefit from the good convergence properties of the finite weight quadratic penalty function and the strict constraint imposed by the Lagrange multiplier. Therefore, the above optimization problem becomes the following unconstrained one:

$$
\begin{aligned}
\mathcal{L}\left(\left\{u_{k}\right\},\right. & \left.\left\{\omega_{k}\right\}, \lambda\right):= \\
& \alpha \sum_{k}\left\|\partial_{t}\left[\left(\delta(t)+\frac{j}{\pi t}\right) * u_{k}(t)\right] e^{-j \omega_{k} t}\right\|_{2}^{2} \\
& +\left\|f(t)-\sum_{k} u_{k}(t)\right\|_{2}^{2}+\left\langle\lambda(t), f(t)-\sum_{k} u_{k}(t)\right\rangle .
\end{aligned}
$$

The alternate direction method of multipliers (ADMM) which is shown in figure 2 is then used to solve the minimization problem of equation (5) by finding the saddle point of the augmented Lagrangian $\mathcal{L}$ in a series of iterative sub-optimizations.

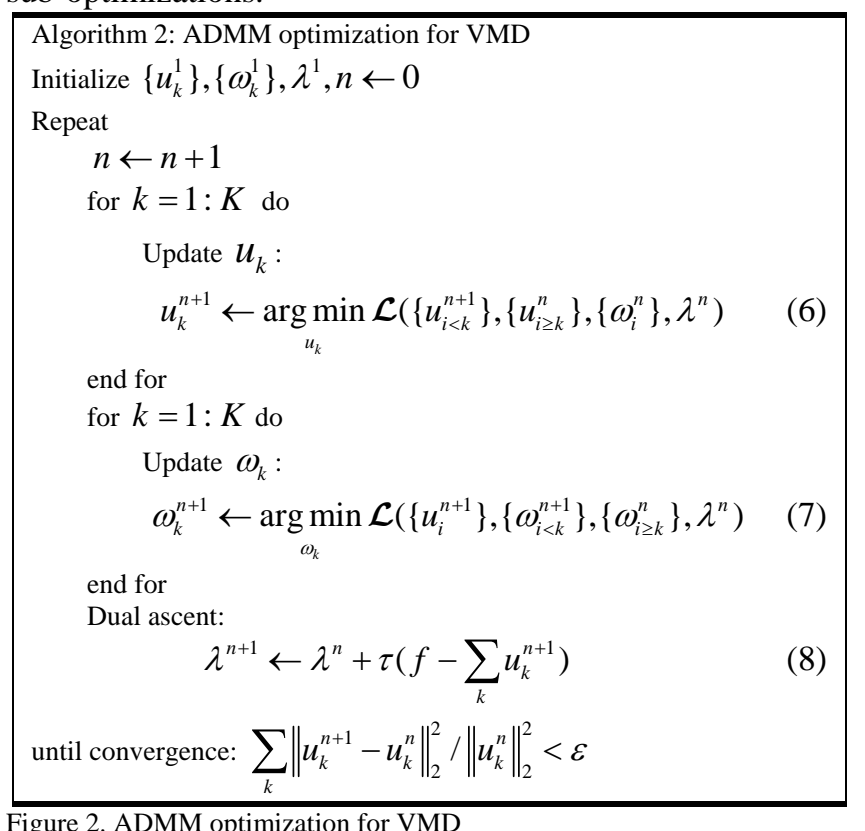

According to ADMM, the VMD process should be implemented by updating and minimizing $u_{k}$ and $\omega_{k}$. The iterative process is as follows:

$$
\begin{gathered}
\hat{u}_{k}^{n+1}(\omega)=\frac{\hat{f}(\omega)-\sum_{i \neq k} \hat{u}_{i}(\omega)+\frac{\hat{\lambda}(\omega)}{2}}{1+2 \alpha\left(\omega-\omega_{k}^{n}\right)^{2}} \\
\omega_{k}^{n+1}=\frac{\int_{o}^{\infty} \omega\left|\hat{u}_{k}^{n+1}(\omega)\right|^{2} d \omega}{\int_{o}^{\infty}\left|\hat{u}_{k}^{n+1}(\omega)\right|^{2} d \omega}
\end{gathered}
$$

Where $\mathrm{n}$ represents the number of iterations, $\hat{f}(\omega), \hat{u}_{k}^{n+1}(\omega), \hat{\lambda}(\omega), \hat{u}_{i}(\omega)$ represent the Fourier transform of $f(t), u_{k}^{n+1}(t), \lambda(t), u_{i}(t)$ respectively.

\section{Wind SPEED PREDICTION MODEL BASED ON VMD AND LORENZ DISTURBANCE}

Based on the Lorenz chaotic system and VMD introduced in the section II, this section will introduce the establishment process of the wind speed prediction model, including the details of the original wind speed series, the setting of model parameters, the prediction process and evaluation index.

\section{A. Original wind speed datasets}

The wind speed data came from the Sotavento wind farm in Galicia, Spain in 2018 [39]. A total of 4 data sets were selected. The starting points were 0:00 on January 2, 0:00 on April 2, 0 on July 2 and 0 on October 2, respectively. Time 
interval is 10 minutes, the first 900 data (about 6 days and 6 hours) are used as training set, and the last 100 data (about
16.7 hours) are used as test set. Figure 3 shows the original wind speed sequence for the four data sets.

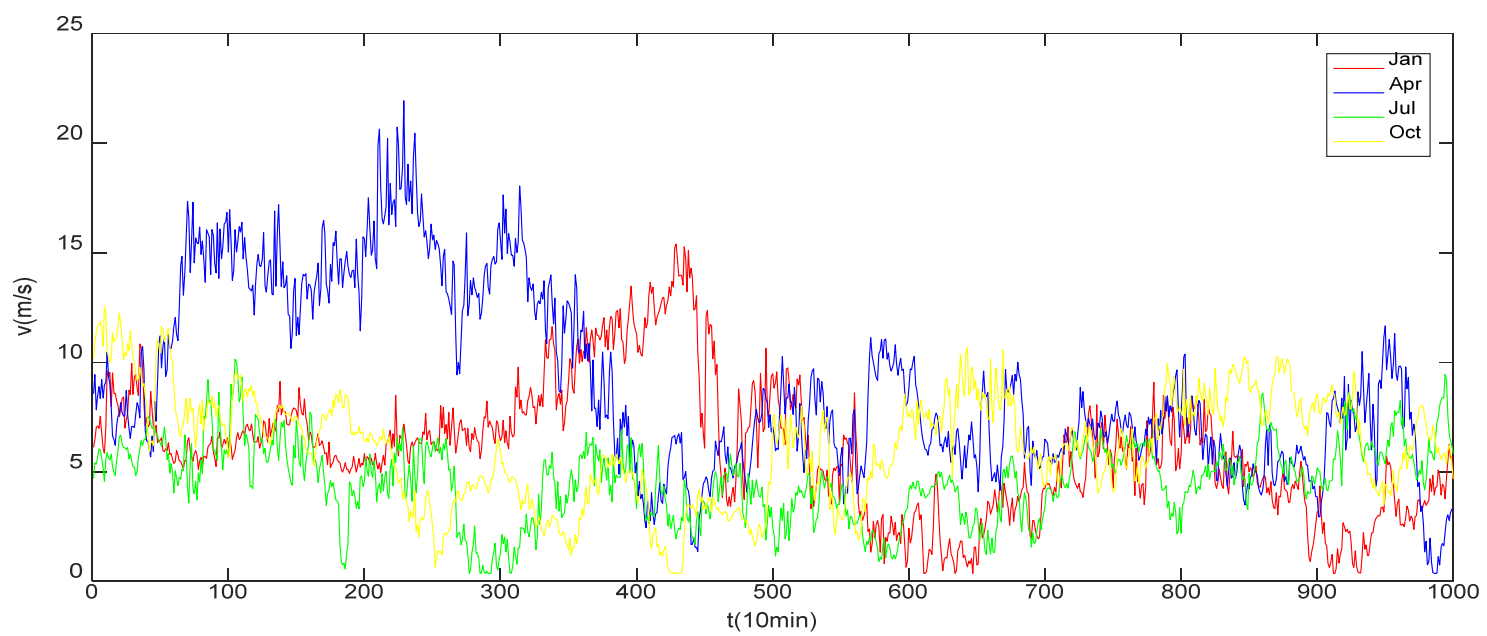

Figure 3. Original wind speed series

As shown in figure 3 , the blue line representing wind speed in April has a large value on the whole. It has an average value of $9.023 \mathrm{~m} / \mathrm{s}$ and a maximum value of 21.93 $\mathrm{m} / \mathrm{s}$. It indicates that the spring wind speed reached the highest level in the year. The red line representing wind speed in January and the yellow line representing wind speed in October are at medium levels among 4 lines. Their average values are $6.04 \mathrm{~m} / \mathrm{s}$ and $6.14 \mathrm{~m} / \mathrm{s}$, respectively. The average value of the green line representing the wind speed in July is $4.61 \mathrm{~m} / \mathrm{s}$, which is the lowest of the four data sets.

\section{B. parameter setting}

For the models involved in this paper, their parameter settings are given in Table I, Table II and Table III.

TABLE I. PARAMETER SETTING OF LORENZ DISTURBANCE

\begin{tabular}{|c|c|}
\hline Parameter & Value \\
\hline initial value of $(\mathrm{x}, \mathrm{y}, \mathrm{z})$ & $(1.1,1,1)$ \\
\hline$\sigma$ & 10 \\
\hline$b$ & $8 / 3$ \\
\hline$r$ & 28 \\
\hline
\end{tabular}

TABLE II. PARAMETER SETTING OF VMD

\begin{tabular}{|c|c|}
\hline Parameter & Value \\
\hline Moderate bandwidth constraint $\alpha$ & 2000 \\
\hline Noise-tolerance $\tau$ & 0 \\
\hline Modes number & 3 \\
\hline
\end{tabular}

TABLE III. PARAMETER SETTING OF DiFFERENT NEURAL NETWORKS

\begin{tabular}{|c|c|c|c|c|}
\hline Parameter & BP & RBF & Elman & LSTM \\
\hline Layer number & 3 & 3 & 4 & 3 \\
\hline $\begin{array}{c}\text { Number of } \\
\text { hidden Layer } \\
\text { neurons }\end{array}$ & 3 & 3 & 3 & 18 \\
\hline $\begin{array}{c}\text { Activation } \\
\text { function }\end{array}$ & $\begin{array}{c}\text { Logsig, } \\
\text { purelin }\end{array}$ & $\begin{array}{c}\text { Gauss } \\
\text { function, } \\
\text { purelin }\end{array}$ & $\begin{array}{c}\text { Logsig, } \\
\text { purelin }\end{array}$ & Sigmoid, tanh \\
\hline Training function & tranlm & $\backslash$ & tranlm & $\begin{array}{c}\text { back- } \\
\text { propagation } \\
\text { through time }\end{array}$ \\
\hline
\end{tabular}

\section{Prediction process}

Based on the theories of Lorenz chaotic system and VMD introduced in section II, we give the process of selecting and establishing wind speed prediction model in this part.

Step 1. The initial Lorenz values, the parameters in Table 1 and the first 900 original wind speed series are brought into the Lorenz equation, thus the Lorenz disturbance coefficient is obtained by solving the equation.

Step 2. Enter the parameters in Table 2 and the first 900 original wind speed series into VMD, The decomposition sequences $u_{i}(i=1,2,3)$ is obtained by computer iteration under the condition of tolerance less than $1 * 10^{-7}$ according to equation (2). Decomposition results are shown in figure 4.

Step 3. Setting the parameters of each neural network according to Table 3. The first 900 numbers of each subsequence serve as training sets for neural networks, and the next 100 serve as test sets.

Step 4. For each data set, each sequence are predicted by BP neural network, RBF neural network, Elman neural network and LSTM neural network, then the prediction results of the same prediction model with different subsequences from the same data set are added up, thus the prediction results of VMD-BP, VMD-RBF, VMD-Elman and VMD-LSTM are obtained.

Step 5. The Lorenz disturbance coefficient obtained in step 1 is directly added to the preliminary prediction results obtained in step 4, thus the preliminary prediction results are corrected and prediction models LD-VMD-BP, LD-VMDRBF, LD-VMD-Elman and LD-VMD-LSTM are obtained. The flowchart of model establishment is shown in Figure 5.

\section{Model evaluation and comparison index}

The commonly used evaluation indexes of the model's prediction accuracy are mean absolute error (MAE), root mean square error (RMSE), and mean absolute percentage error (MAPE). In this paper we use MAE and RMSE to evaluate the prediction effect. The specific calculation formulas are as follows:

$$
\begin{aligned}
M A E & =\frac{1}{n} \sum_{t=1}^{n}|y(t)-\hat{y}(t)| \\
R M S E & =\sqrt{\frac{1}{n} \sum_{t=1}^{n}(y(t)-\hat{y}(t))^{2}}
\end{aligned}
$$

Where $y(t)$ denotes original wind speed, $\hat{y}(t)$ denotes wind speed predictions, $n$ indicates the sample size.

We first compare the MAE and RMSE of different models on the same dataset to obtain the model with the highest prediction accuracy, and then carry out experimental verification on multiple datasets to avoid the exceptions 
caused by a single dataset.

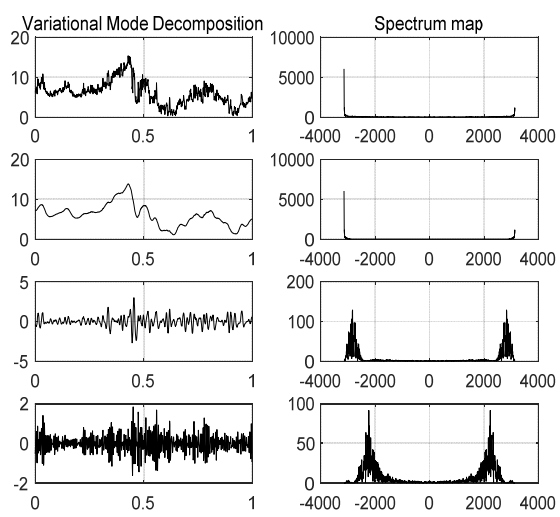

(a) VMD decomposition result of 1000 wind speeds in January
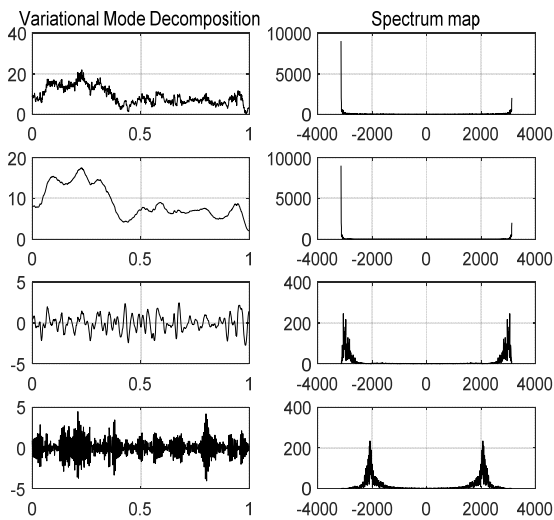

(b) VMD decomposition result of 1000 wind speeds in April
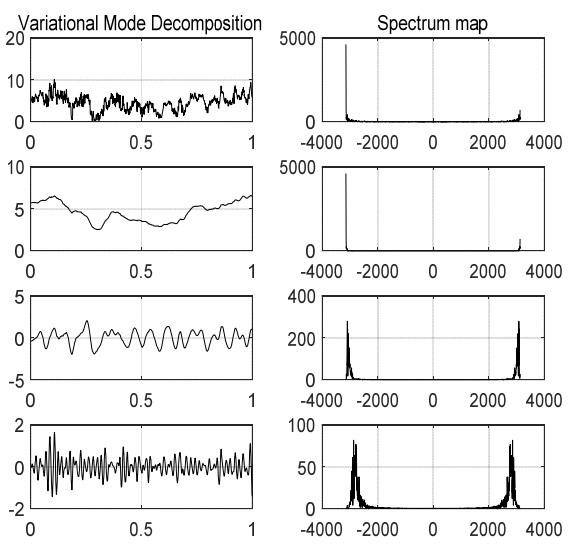

(c) VMD decomposition result of 1000 wind speeds in July
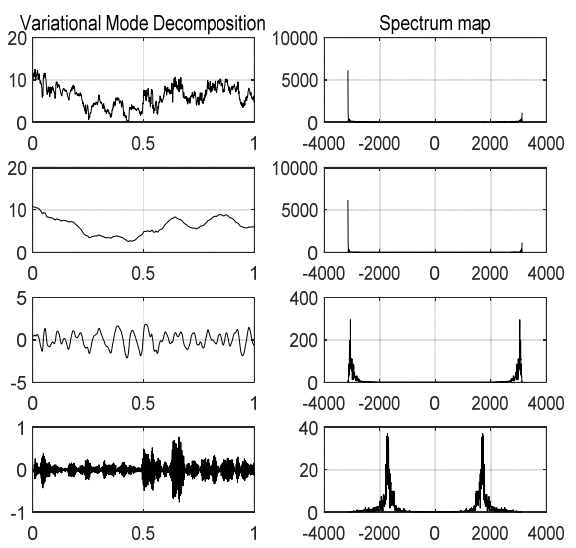

(d) VMD decomposition result of 1000 wind speeds in October

Figure 4. 1000 wind speed VMD decomposition result on 4 data sets

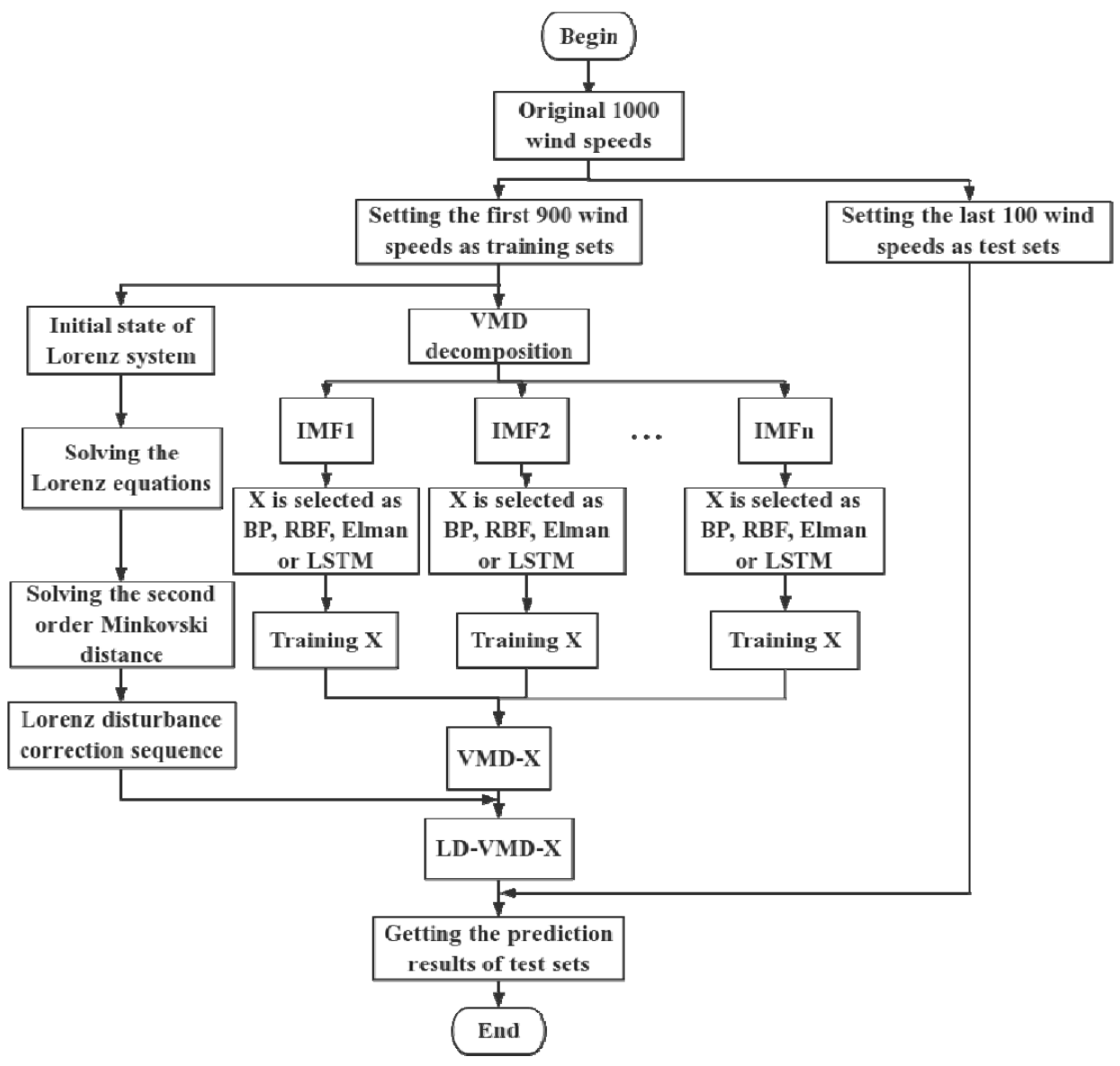

Figure 5. Flow chart of model building 


\section{EXPERIMENTAL RESUlts AND ERROR ANALYSIS}

After introducing the process of model establishing, in this section we simulate different models on several data sets. Firstly, on the wind speed data set of January 2018, VMD and Lorenz disturbance are used to improve various prediction models in turn, and verifying the effect of VMD and Lorenz disturbance on model predictions. Then, by comparing MAE and RMSE, the best prediction model is obtained. Finally, the model is tested on multiple data sets to verify its universality.

A. Preliminary comparison between neural network models and other models

We first compare 4 neural networks with the persistence method, the ARMA prediction model, and the SVM prediction model. The prediction results on the four data sets are shown in Figure 6, and their MAE and RMSE are calculated separately as shown in Table IV.

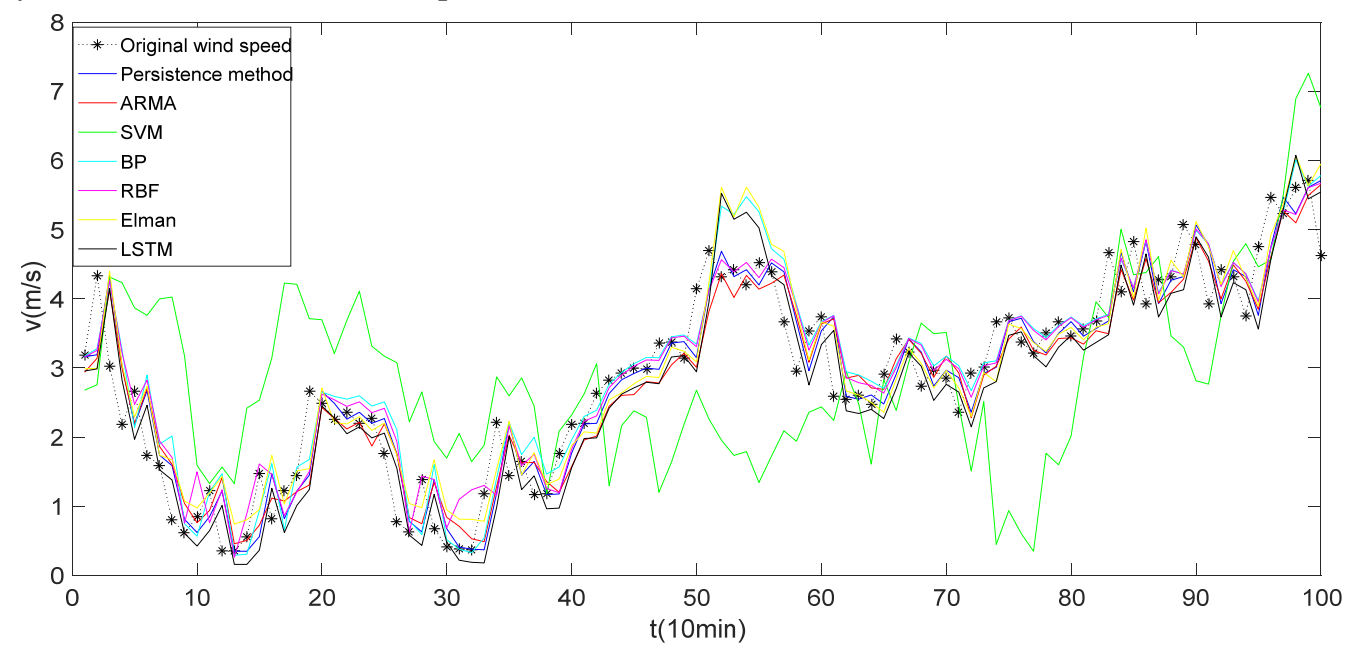

(a) Prediction results of persistence method, ARMA, SVM, BP, RBF, Elman and LSTM on January

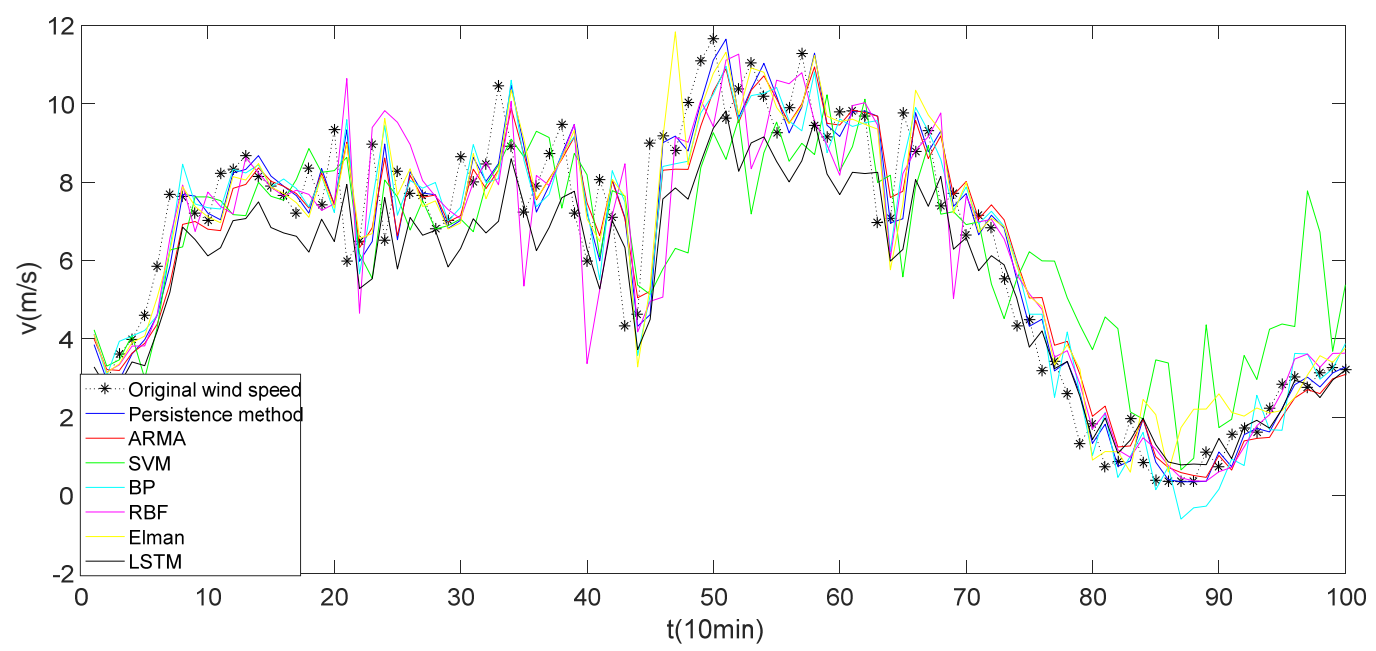

(b) Prediction results of persistence method, ARMA, SVM, BP, RBF, Elman and LSTM on April

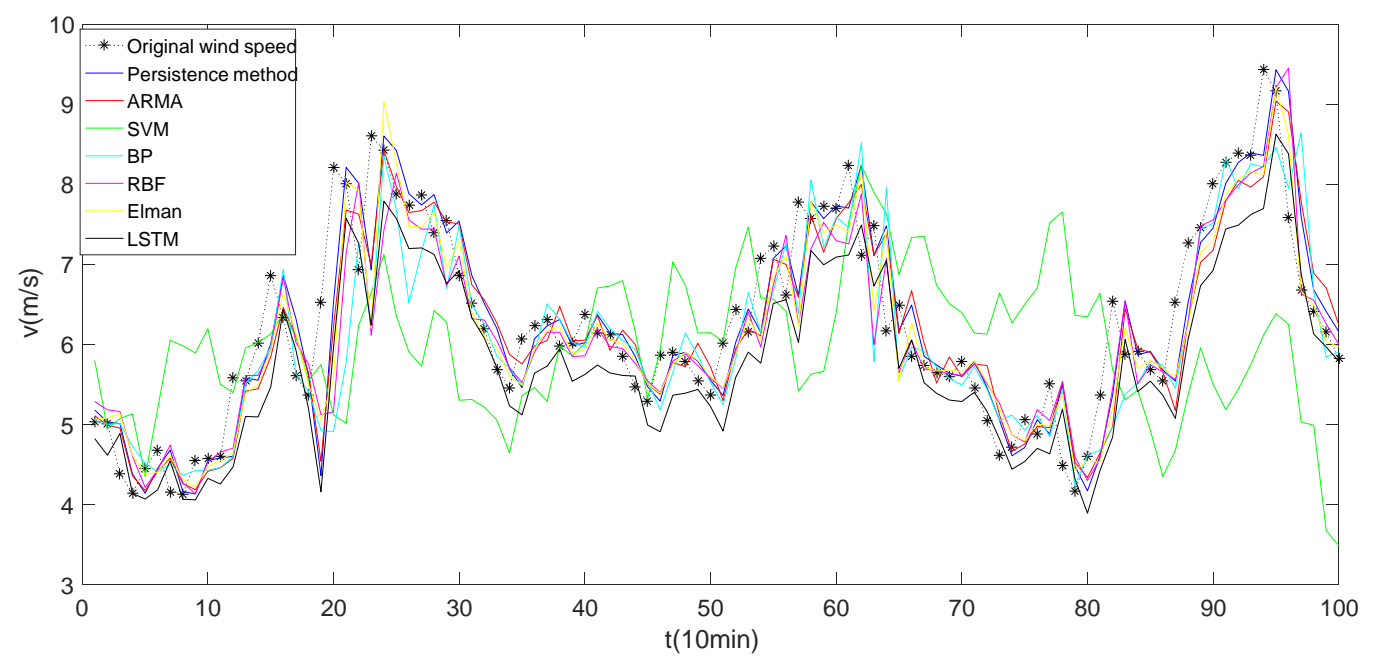

(c) Prediction results of persistence method, ARMA, SVM, BP, RBF, Elman and LSTM on July 


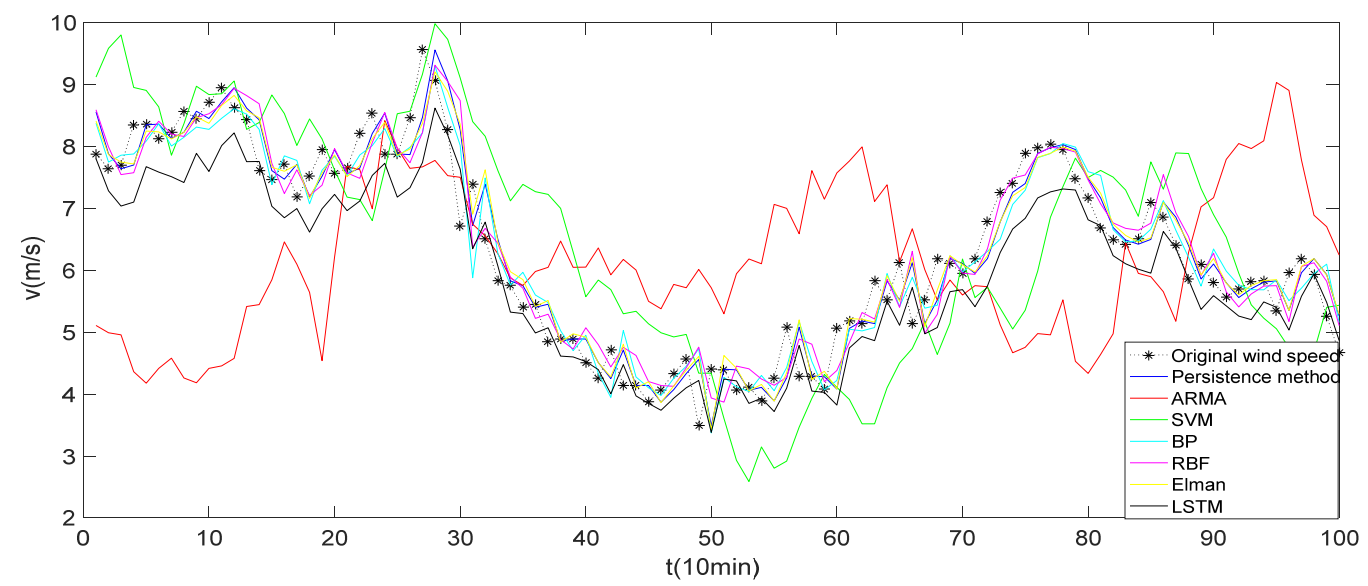

(d) Prediction results of persistence method, ARMA, SVM, BP, RBF, Elman and LSTM on October

Figure 6. (a)-(d) Separately depict prediction results of persistence method, ARMA, SVM, BP, RBF, Elman and LSTM on 4 different datasets

TABLE IV. MAE AND RMSE OF PERSISTENCE METHOD, ARMA, SVM, BP, RBF, ELMAN AND LSTM ON FOUR DATA SETS

\begin{tabular}{|c|c|c|c|c|c|}
\hline & & January & April & July & October \\
\hline \multirow{7}{*}{ MAE } & persistence method & 0.4847 & 0.8969 & 0.4488 & 0.3779 \\
\hline & ARMA & 0.4918 & 1.2213 & 0.4771 & 1.8384 \\
\hline & SVM & 1.2939 & $\overline{1.4792}$ & 1.2116 & 1.0099 \\
\hline & $\mathrm{BP}$ & 0.4953 & 0.9697 & 0.5749 & 0.3873 \\
\hline & $\mathrm{RBF}$ & 0.4733 & 1.0496 & 0.4993 & 0.4147 \\
\hline & Elman & 0.4767 & 1.0014 & 0.4604 & 0.3801 \\
\hline & LSTM & 0.4951 & 1.1667 & 0.5797 & 0.5271 \\
\hline \multirow{7}{*}{ RMSE } & persistence method & 0.6034 & 1.2121 & 0.6104 & 0.4800 \\
\hline & ARMA & 0.6180 & 1.5855 & 0.6359 & 2.1640 \\
\hline & SVM & 1.5369 & 1.8688 & 1.4638 & 1.1946 \\
\hline & $\mathrm{BP}$ & $\overline{0.6285}$ & $\overline{1.2826}$ & $\overline{0.8794}$ & $\overline{0.5038}$ \\
\hline & $\mathrm{RBF}$ & 0.5975 & 1.4013 & 0.7083 & 0.5262 \\
\hline & Elman & 0.6029 & 1.2931 & 0.6552 & 0.4865 \\
\hline & LSTM & 0.6091 & 1.4775 & 0.7660 & 0.6480 \\
\hline
\end{tabular}

The predictable time of the persistence method is too short (usually within one hour and in this paper is 10 minutes), so its application is limited. In this paper, it is only used as a comparison standard to measure the prediction effect of other prediction models. And from Figure 6 and Table IV we can see that SVM performs worst in all data sets. On April dataset and October dataset, the MAE and RMSE of ARMA and SVM are both much larger than those of other models (these data are underlined in the table). 4 neural networks and persistence methods perform well on every data set. Therefore, the prediction effect of the neural network wind speed prediction model on any data set is obviously better than that of other comparative models.

Next, we will optimize the wind speed prediction model based on 4 neural networks in order to obtain a hybrid model with higher prediction accuracy.

\section{B. Optimal neural network prediction model}

On the wind speed data set of January 2018, based on BP, RBF, Elman and LSTM prediction models, we optimized them and corrected the predictions respectively. Figure 7 shows the prediction results.

In Fig. 7, (a) is the prediction results of $\mathrm{BP}$ neural network and its optimized models, (b) is that of RBF neural network and its optimized models, (c) is that of Elman neural network and its optimized models, (d) is that of LSTM neural network and its optimized models. The black fold lines are the original wind speed, blue lines are the prediction results of $\mathrm{X}$ (X denotes one of BP, RBF, Elman and LSTM), red lines represent the prediction results of
VMD-X, green lines are the results of LD-VMD-X.

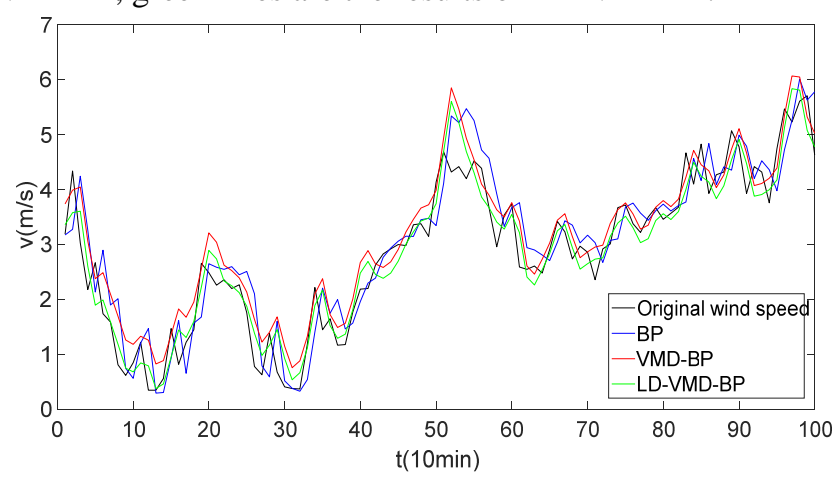

(a) Prediction results based on BP

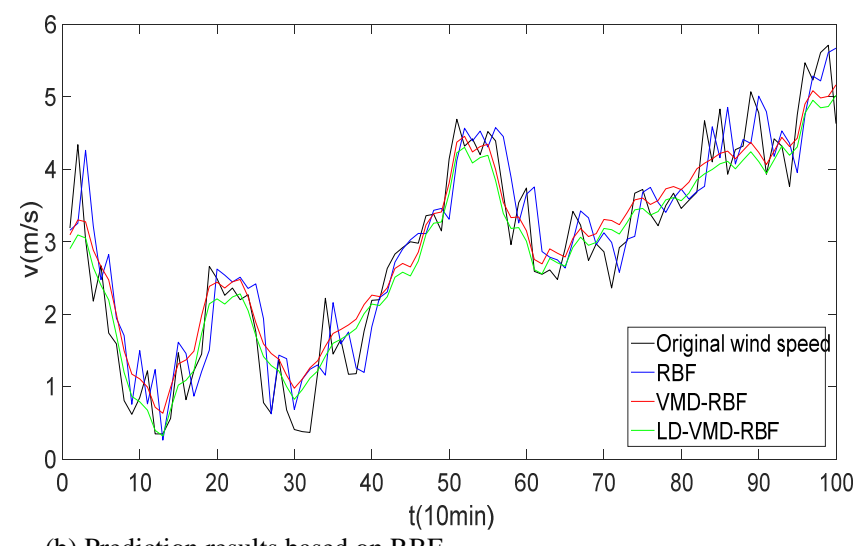

(b) Prediction results based on RBF 


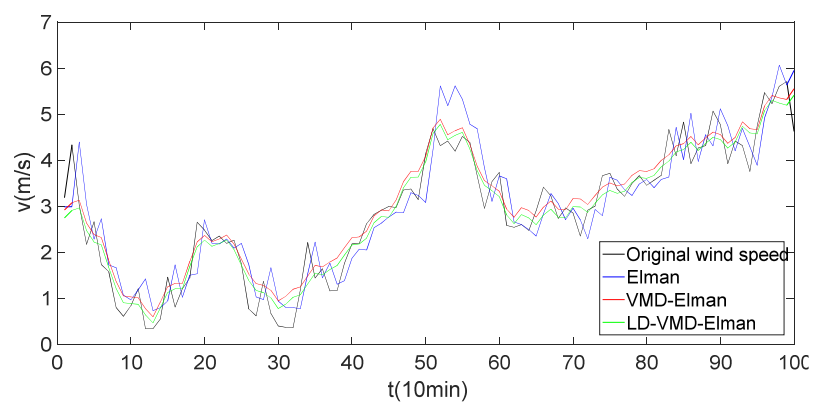

(c) Prediction results based on Elman

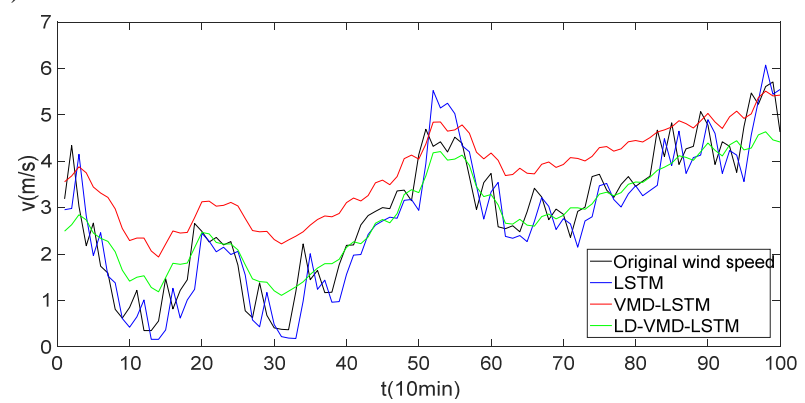

(d) Prediction results based on LSTM

Figure 7. (a) - (d) Separately depict wind speed prediction result on January based on BP, RBF, Elman, LSTM

It can be seen from figure 7 that the one-step hysteresis of prediction results of the single model is obvious. After adding VMD, the hysteresis is obviously weakened, and the prediction result is closer to real wind speed. After imposing Lorenz perturbation correction on VMD-X, the green line of LD-VMD-X is the nearest to original wind speed.

We calculated MAE and RMSE for 12 models (BP, VMD-BP, LD-VMD-BP, RBF, VMD- RBF, LD-VMDRBF, Elman, VMD-Elman, LD-VMD-Elman, LSTM, VMD-LSTM and LD-VMD-LSTM), the results as shown in Table V.
In Table $\mathrm{V}$, for the BP, RBF, Elman, and LSTM prediction models, both MAE and RMSE are significantly reduced after adding VMD and Lorenz disturbance. Moreover, regardless of MAE or RMSE, it is the smallest of LD-VMD-Elman, which is 0.2872 and 0.3717 respectively. This shows that LD-VMD-Elman's prediction effect is the best in Spain's January 2018 wind speed data set. Compared to Elman, its MAE was reduced by $39.8 \%$ and RMSE was reduced by $38.3 \%$. Compared to VMD-Elman, its MAE was reduced by $10.1 \%$ and RMSE was reduced by $7.1 \%$.

\section{LD-VMD-Elman's universality}

We experimented with the best-performing LD-VMDElman model on multiple datasets to verify the universality of the model. The prediction results are shown in Figure 8.

In Figure 8, (a) shows the prediction results of Elman, VMD-Elman and LD-VMD-Elman on January wind speed data set (representing winter), (b) is that on April wind speed dataset (representing spring), (c) is that on July wind speed data set (representing summer), (d) is that on October wind speed data set (representing autumn). The black lines are the original wind speed, the blue lines represent Elman prediction results, the red lines represent VMD-Elman prediction results, and the green lines represent LD-VMDElman prediction results.

As can be seen from figure 8, regardless of which data set, the prediction results of the VMD-Elman model are much closer to the true wind speed sequence than Elman's. After the Lorenz disturbance correction, the prediction result of the LD-VMD-Elman model further improves the prediction accuracy. The green line representing LD-VMDElman is the closest line to the original wind speed line which is black on any sub-graph.

TABLE V. MAE AND RMSE OF EACH MODEL'S PREDICTED RESULTS ON JANUARY

\begin{tabular}{|l|l|l|l|}
\hline \multirow{2}{*}{ Prediction modes } & \multicolumn{2}{c|}{ Error } \\
\cline { 2 - 4 } & BP & MAE & 0.5885 \\
\hline \multirow{4}{*}{ Modes based on BP } & VMD-BP & 0.4553 & 0.4909 \\
\cline { 2 - 4 } & LD-VMD-BP & 0.3869 & 0.3900 \\
\hline \multirow{4}{*}{\begin{tabular}{l} 
Modes based on RBF \\
\cline { 2 - 4 }
\end{tabular}} & RBF & 0.3162 & 0.5375 \\
\cline { 2 - 4 } \begin{tabular}{l} 
Elman based on \\
\cline { 2 - 4 }
\end{tabular} & VMD- RBF & 0.4133 & 0.4098 \\
\cline { 2 - 4 } & LD-VMD- RBF & 0.3323 & 0.3977 \\
\cline { 2 - 4 } & Elman & 0.3104 & 0.6029 \\
\cline { 2 - 4 } $\begin{array}{l}\text { Modes based on } \\
\text { LSTM }\end{array}$ & VMD- Elman & 0.4767 & 0.4001 \\
\cline { 2 - 4 } & LS-VMD- Elman & 0.3194 & $\mathbf{0 . 3 7 1 7}$ \\
\cline { 2 - 4 } & VMD- LSTM & $\mathbf{0 . 2 8 7 2}$ & 0.6091 \\
\cline { 2 - 4 } & LD-VMD- LSTM & 0.4951 & 0.5801 \\
\hline
\end{tabular}

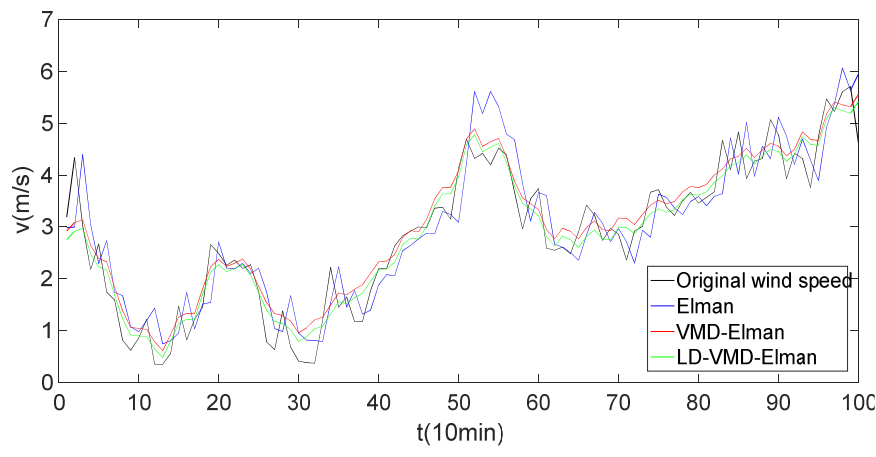

(a) Prediction results based on Elman on January

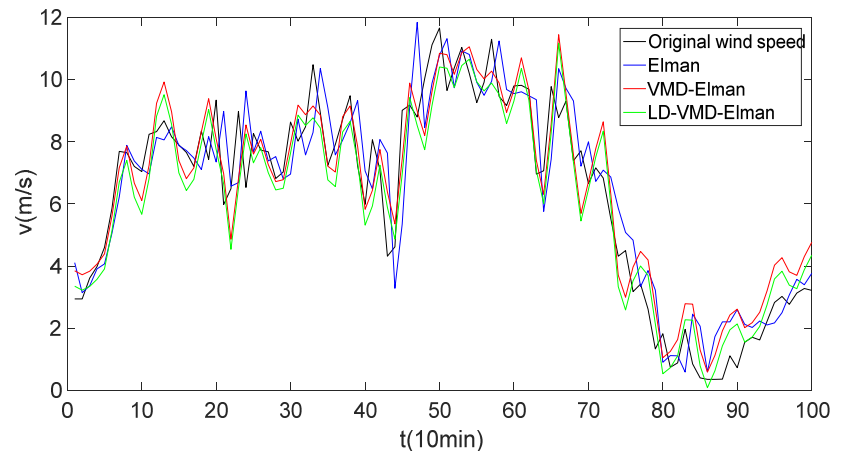

(b) Prediction results based on Elman on March 


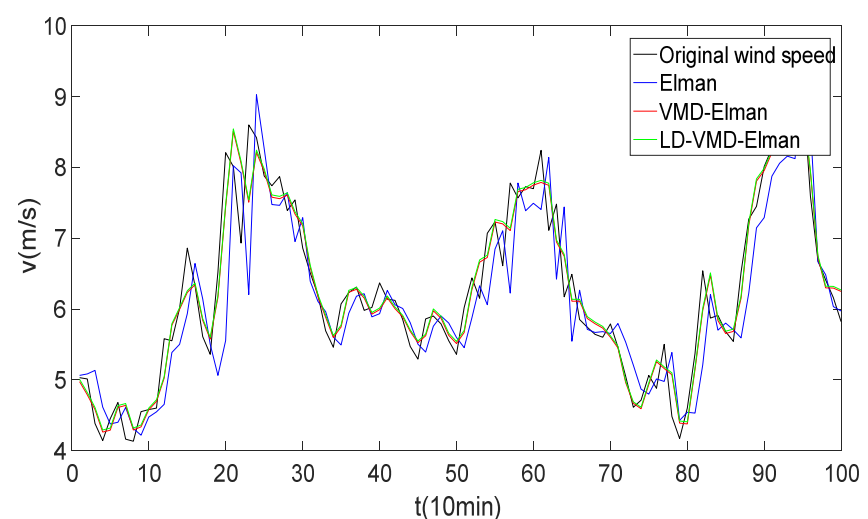

(c) Prediction results based on Elman on July

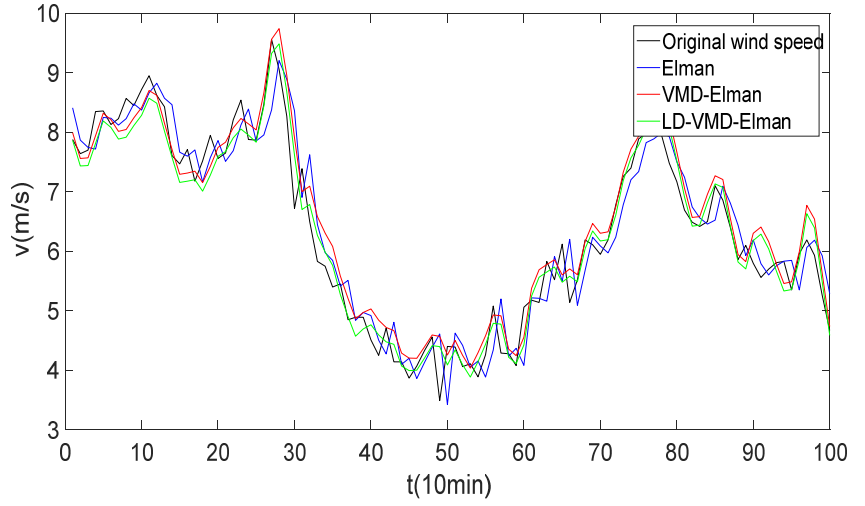

(d) Prediction results based on Elman on October

Figure 8. (a)-(d) Separately depict prediction results of Elman, VMD-Elman and LD-VMD-Elman on 4 different data sets

TABLE VI. MAE AND RMSE OF ELMAN, VMD-ELMAN AND LD-VMD-ELMAN ON FOUR DATA SETS

\begin{tabular}{|l|l|l|l|}
\hline \multicolumn{2}{|c|}{} & MAE & RMSE \\
\hline \multirow{4}{*}{ January } & Elman & 0.4767 & 0.6029 \\
\cline { 2 - 4 } & VMD- Elman & 0.3194 & 0.4001 \\
\cline { 2 - 4 } & LD-VMD- Elman & 0.2872 & 0.3717 \\
\hline \multirow{4}{*}{ July } & Elman & 1.0014 & 1.2931 \\
\cline { 2 - 4 } & VMD- Elman & 0.8416 & 1.0285 \\
\cline { 2 - 4 } & LD-VMD- Elman & 0.8011 & 1.0107 \\
\hline \multirow{3}{*}{ October } & Elman & 0.4604 & 0.6552 \\
\cline { 2 - 4 } & VMD- Elman & 0.2510 & 0.3410 \\
\cline { 2 - 4 } & LD-VMD- Elman & $\mathbf{0 . 2 4 1 3}$ & $\mathbf{0 . 3 3 0 9}$ \\
\cline { 2 - 4 } & Elman & 0.3801 & 0.4865 \\
\cline { 2 - 4 } & VMD- Elman & 0.3015 & 0.3849 \\
\cline { 2 - 4 } & LD-VMD- Elman & 0.2622 & 0.3335 \\
\hline
\end{tabular}

We calculated the MAE and RMSE for the prediction results of Elman, VMD-Elman, and LD-VMD-Elman on the four data sets, the results as shown in Table VI.

In Table VI, VMD-Elman's MAE and RMSE are smaller than Elman's, and LD-VMD-Elman's MAE and RMSE are smaller than VMD-Elman's. This shows that the VMD and Lorenz disturbances can improve the accuracy of the wind speed prediction model, which is true on all data sets. More specifically, LD-VMD- Elman's MAE is 0.1817 less than Elman's MAE, and the RMSE is reduced by an average of 0.2477 on four data sets. The smallest MAE and RMSE appear on the July data set.

\section{CONCLUSION}

Accurate wind speed prediction is crucial for power dispatch planning, maintenance scheduling and regulation. Therefore, this paper proposes the LD-VMD-Elman wind speed prediction model. First, a set of Lorenz equations is used to describe the atmospheric dynamic system, and the equation is solved under given initial conditions and parameters to obtain the optimal disturbance coefficient. Secondly, the original wind speed sequence is decomposed by VMD to obtain IMFs. Then, considering the characteristics of neural network that can deal with highly non-linear time series, the IMF components are predicted by BP, RBF, Elman and LSTM neural network. Finally, the optimal disturbance coefficient obtained in the first step is used to correct the prediction result. And compared with other models, the LD-VMD-Elman wind speed prediction model with the highest prediction accuracy is obtained. Simulation results show that the addition of VMD and
Lorenz disturbance can significantly improve the prediction accuracy of the initial model, LD-VMD-Elman is the most accurate prediction model in 12 wind speed prediction models. The contribution of this paper is that the atmospheric dynamic system is considered into the wind speed prediction model, and the wind speed prediction results are modified by Lorenz disturbance. After combining VMD and different neural networks, the LD-VMD-Elman wind speed prediction model proposed in this paper can improve the prediction accuracy by up to $49.5 \%$ compared with the other 11 models. It does contribute to the largescale development and utilization of wind energy.

\section{REFERENCES}

[1] E. Ssekulima, M. B. Anwar, A. A. Hinai, M. S. Elmoursi, "Wind speed and solar irradiance forecasting techniques for enhanced renewable energy integration with the grid: a review," IET Renewable Power Generation, vol. 10, no. 7, pp. 885-898, 2016. doi:10.1049/ietrpg.2015.0477

[2] J. Zhao, Y. L. Guo, X. Xiao, J. Z. Wang, D. Z. Chi, Z. H. Guo, "Multi-step wind speed and power forecasts based on a WRF simulation and an optimized association method," Applied Energy, vol. 197, pp. 183-202, 2017. doi:10.1016/j.apenergy.2017.04.017

[3] W. Y. Y. Cheng, Y. B. Liu, Y. W. Liu, Y. X. Zhang, W. R. Mahoney, T. T. Warner, "The impact of model physics on numerical wind forecasts,” Renewable Energy, vol. 55, pp. 347-356, 2013. doi:10.1016/j.renene.2012.12.041

[4] J. Zhao, Z. H. Guo, Z. Y. Su, Z. Y. Zhao, X. Xiao, F. Liu, “An improved multi-step forecasting model based on WRF ensembles and creative fuzzy systems for wind speed,” Applied Energy, vol. 162, pp. 808-82, 2016. doi:10.1016/j.apenergy.2015.10.145

[5] Y. G. Zhang, Y. Zhao, G. F. Pan, J. F. Zhang, "Wind speed interval prediction based on Lorenz disturbance distribution," IEEE Transactions on Sustainable Energy, doi:10.1109/TSTE.2019.2907699 
[6] E. Erdem, J. Shi, “ARMA based approaches for forecasting the tuple of wind speed and direction,” Applied Energy, vol. 88, pp. 14051414, 2011. doi:10.1016/j.apenergy.2010.10.031

[7] J. Bessac, E. Mihai Constantinescu, M. Anitescu, "Stochastic simulation of predictive space-time scenarios of wind speed using observations and physical models,” Annals of Applied Statistics, vol. 12, no.1, pp. 432-45, 2018. doi:10.1214/17-AOAS1099

[8] P. Ramasamy, S. S. Chandel, A. K. Yadav, "Wind speed prediction in the mountainous region of India using an artificial neural network model,” Renewable Energy, vol. 80, pp. 338-347, 2015. doi:10.1016/j.renene.2015.02.034

[9] G. Song, Q. Dai, “A novel double deep ELMs ensemble system for time series forecasting,” Knowledge-Based Systems, vol. 134, pp. 3149, 2017. doi:10.1016/j.knosys.2017.07.014

[10] H. Liu, H. Q. Tian, X. F. Liang, Y. F. Li, “Wind speed forecasting approach using secondary composition algorithm and Elman neural networks,” Applied Energy, vol. 157, pp. 183-194, 2015. doi:10.1016/j.apenergy.2015.08.014

[11] Z. S. Yang, J. Wang, “A hybrid forecasting approach applied in wind speed forecasting based on a data processing strategy and an optimized artificial intelligence algorithm,” Energy, vol. 160, pp. 87100, 2018. doi:10.1016/j.energy.2018.07.005

[12] J. Z. Wang, S. H. Xiong, "A hybrid forecasting model based on outlier detection and fuzzy time series - A case study on Hainan wind farm of China,” Energy, vol. 76, pp. 526-541, 2014. doi:10.1016/j.energy.2014.08.064

[13] O. Karakus, E. E. Kuruoglu, M. A. Altinkaya, "One-day ahead wind speed/power prediction based on polynomial autoregressive model," IET Renewable Power Generation, vol. 11, no. 11, pp. 1430-1439, 2017. doi:10.1049/iet-rpg.2016.0972

[14] Y. G. Zhang, B. Chen, G. F. Pan, Y. Zhao, "A novel hybrid model based on VMD-WT and PCA-BP-RBF neural network for short-term wind speed forecasting," Energy Conversion and Management, vol. 195, pp. 180-197, 2019.doi: 10.1016/j.enconman.2019.05.005

[15] Q. H. Hu, S. G. Zhang, M. Yu, Z. X. Xie, "Short-term wind speed or power forecasting with heteroscedastic support vector regression," IEEE Transactions on Sustainable Energy, vol.7, pp. 241-249, 2016. doi:10.1109/TSTE.2015.2480245

[16] X. B. Kong, X. J. Liu, R. F. Shi, K. Y. Lee, "Wind speed prediction using reduced support vector machines with feature selection," Neurocomputing, vol. 169, pp. 449-456, Dec. 2015. doi:10.1016/j.neucom.2014.09.090

[17] Y. Ren, P. N. Suganthan, N. Srikanth, "A novel empirical mode decomposition with support vector regression for wind speed forecasting," IEEE Transactions on Neural Networks and Learning Systems, vol. 27, no. 8, pp. 1793-1798, 2016 doi:10.1109/TNNLS.2014.2351391

[18] C. J. Yu, Y. L. Li, Y. L. Bao, H. J. Tang, G. H. Zhai, “A novel framework for wind speed prediction based on recurrent neural networks and support vector machine," Energy Conversion and Management, vol. 178, pp. 137-145, 2018. doi:10.1016/j.enconman.2018.10.008

[19] P. Jiang, Y. Wang, J. Z. Wang, "Short-term wind speed forecasting using a hybrid model,” Energy, vol. 119, pp. 561-577, 2017. doi:10.1016/j.energy.2016.10.040

[20] Y. G. Zhang, C. H. Zhang, S. Gao, P. H. Wang, F. L. Xie, P. L. Cheng, S. Lei, "Wind speed prediction using wavelet decomposition based on Lorenz disturbance model," IETE Journal of Research. doi:10.1080/03772063.2018.1512384

[21] Y. H. Chen, Z. S. He, Z. H. Shang, C. H. Li, L. Li, M. L. Xu, “A novel combined model based on echo state network for multi-step ahead wind speed forecasting: A case study of NREL," Energy Conversion and Management, vol. 179, pp. 13-29, 2019. doi:10.1016/j.enconman.2018.10.068
[22] C. J. Huang, P. H. Kuo, "A Short-term wind speed forecasting mode by using artificial neural networks with stochastic optimization for renewable energy systems,” Energies, vol. 11, no. 10, 2018. doi:10.3390/en11102777

[23] Y. G. Zhang, Y. Zhao, S. Gao, “A novel hybrid model for wind speed prediction based on VMD and neural network considering atmospheric uncertainties,” IEEE Access. doi:10.1109/ACCESS.2019.2915582

[24] Y. G. Zhang, P. H. Wang, T. Ni, P. L. Cheng, S. Lei. "Wind power prediction based on LS-SVM model with error correction,” Advances in Electrical and Computer Engineering, vol. 17, pp.3-8, Jan. 2017. doi:10.4316/AECE.2017.01001

[25] L. L. Wang, X. Li, Y. L. Bai, "Short -term wind speed prediction using an extreme learning machine model with error correction," Energy Conversion and Management, vol. 162, pp. 239-250, 2018. doi:10.1016/j.enconman.2018.02.015

[26] E. N. Lorenz, "Nondeterministic theories of climatic change," Quaternary Research, pp.495-506, 1976. doi:10.1016/00335894(76)90022-3

[27] Y. G. Zhang, P. H. Wang, P. L. Cheng, S. Lei, "Wind speed prediction with wavelet time series based on Lorenz disturbance," Advances in Electrical and Computer Engineering, vol. 17, pp.107114, 2017. doi:10.4316/AECE.2017.03014

[28] R. Ye, Q. Dai, “A novel transfer learning framework for time series forecasting,” Knowledge-Based Systems, vol. 156, pp. 74-99, 2018. doi:10.1016/j.knosys.2018.05.021

[29] J. Naik, S. Dash, P. K. Dash, R. Bisoi, "Short term wind power forecasting using hybrid variational mode decomposition and multikernel regularized pseudo inverse neural network," Renewable Energy, vol. 118, pp. $180 \quad-212, \quad 2018$. doi:10.1016/j.renene.2017.10.111

[30] J. Medina, M. Ojeda-Aciego. "Multi-adjoint t-concept lattices," Information Sciences, vol. 180, no. 5, pp. 712-725, 2010 doi:10.1016/j.ins.2009.11.018

[31] C. Pozna, R. E. Precup, J. K. Tar, I. Skrjanc, S. Preitl, “New results in modelling derived from Bayesian filtering," Knowlege-Based Systems, vol. 23, no. 2, pp. 182-194, 2010 doi:10.1016/j.knosys.2009.11.015

[32] J. Saadat, P. Moallem, H. Koofigar, "Training echo state neura network using harmony search algorithm,” International Journal of Artificial Intelligence, vol. 15, no. 1, pp. 163-179, 2017.

[33] J. Ruiz-Rangel, C. J. Hernandez, L. M. Gonzalez, D. J. Molinares, "ERNEAD: Training of artificial neural networks based on a genetic algorithm and finite automata theory,” International Journal of Artificial Intelligence, vol. 16, no. 1, pp. 214-253, 2018.

[34] A. Glowacz, "Acoustic-based fault diagnosis of commutator motor," Mechanical Systems and Signal Processing, vol. 7, no. 11, 2018 doi:10.1016/j.ymssp.2018.07.044

[35] A. Glowacz, "Fault diagnosis of single-phase induction motor based on acoustic signals,” Electronics, vol. 117, pp. 65-80, 2019. doi:10.3390/electronics7110299

[36] C. H. Yao, Q. Dai, G. Song, "Several Novel Dynamic Ensemble Selection Algorithms for Time Series Prediction,” Neural Processing Letters, 2018. doi:10.1007/s11063-018-9957-7

[37] K. Dragomiretskiy, D. Zosso, "Variational mode domposition,” IEEE Transactions on Signal Processing, vol. 62, pp. 531-544, 2014 doi:10.1109/TSP.2013.2288675

[38] A. Glowacz, W. Glowacz, "Vibration-Based Fault Diagnosis of Commutator Motor," Shock and Vibration, 2018. doi:10.1155/2018/7460419

[39] The Sotavento wind farm in Galicia, Spain, 2018. [Online]. Available: http://www.sotaventogalicia.com/es/datos-tiempo-real/historicos 